DR RIIKKA TUULI ILONA UOTILA (Orcid ID : 0000-0001-5708-2770)

Article type : Letter to the Editor

\title{
Peanut oral immunotherapy increases IgG4 to Ara h 1, 2, and 6 but does not affect IgG4 to other allergens
}

Short title: Peanut oral immunotherapy increases IgG4 to Ara h 1, 2, and 6

Authors: Riikka Uotila MD ${ }^{1}$, Anna Kaarina Kukkonen $\mathrm{MD}, \mathrm{PhD}^{1}$, Dario Greco $\mathrm{PhD}^{2}$, Anna Susanna Pelkonen MD, $\mathrm{PhD}^{1}$, and Mika Juhani Mäkelä MD, $\mathrm{PhD}^{1}$

${ }^{1}$ Allergology, University of Helsinki and Helsinki University Hospital; the Skin and Allergy Hospital

${ }^{2}$ Faculty of Medicine and Life Sciences, Institute of Biosciences and Medical Technologies (BioMediTech), University of Tampere and Institute of Biotechnology, University of Helsinki

Corresponding author: Riikka Uotila, Skin and Allergy Hospital, Helsinki University Central Hospital, P.O. Box 160, 00029 HUCH, Helsinki, Finland. tel: +358405164560 fax: +358947186416, riikka.uotila@helsinki.fi

Declaration of all sources of funding: the Foundation for Pediatric Research, the Sigrid Juselius Foundation, the Helsinki University Research Funds, the Foundation for Allergy Research, and the Helsinki Allergy Society. Prof Greco was supported by the Academy of Finland (grant agreements 275151 and 292307). ThermoFisher Scientific provided the IgG4 ISAC laboratory analyses. The sponsors had no involvement in the collection, analysis, and interpretation of data; in the writing of the report; or in the decision to submit the article for publication.

This article has been accepted for publication and undergone full peer review but has not been through the copyediting, typesetting, pagination and proofreading process, which may lead to differences between this version and the Version of Record. Please cite this article as doi: 10.1111/pai.13012

This article is protected by copyright. All rights reserved. 


\section{Conflicts of interest: None}

Statement of contribution: Each named author has: 1) substantially contributed to conception and design or acquisition of data, or analysis and interpretation of data; 2) drafting the article or critically revising it for important intellectual content; and 3) finally approved the version to be published.

Trial Registration: clinicaltrials.gov: NCT01502878

\section{KEY WORDS:}

Cross-reactivity, IgG4, Immuno-Solid phase Allergen Chip, Immunotherapy, Peanut allergens

\section{To the Editor:}

Life-threatening allergic reactions are characterized by allergen-specific $\operatorname{IgE}$ antibodies, whereas $\operatorname{IgG}$ antibodies are a default response to an antigen exposure. Subclass $4 \mathrm{IgG}$ antibodies function as blocking antibodies, which may suppress IgE-mediated reactions. ${ }^{1}$ Peanut oral immunotherapy (OIT), as other allergen immunotherapies, induces IgG4 antibodies. ${ }^{2,3}$ We have previously shown the increase of IgG4 to peanut seed storage proteins, and the dose-dependent increase of IgG4- IgE ratios in children and adolescents receiving peanut OIT ${ }^{4,5}$ Further, we showed that IgE to the $2 \mathrm{~S}$ albumins Ara h 2 and Ara h 6, which are the most important peanut allergens, ${ }^{6}$ were the only IgE responses affected during OIT. ${ }^{5}$

In this study, we screened IgG4 responses for a wide spectrum of allergens with the ISAC ImmunoCAP microarray in children and adolescents who underwent peanut OIT. We examined the IgG4 responses baseline and after OIT build-up phase with the aim of finding effects of the treatment on $\mathrm{IgG} 4$ responses to peanut-specific and cross-reactive allergens.

The study population has been described in our previous publications. ${ }^{4,5}$ Briefly, 6- to 18 year-old children and adolescents were referred to the hospital because of peanut allergy. Of them, 39 with a moderate-to-severe reaction in a double-blind placebo-controlled peanut 
challenge started OIT and 21 control patients continued to avoid peanut. During OIT, the daily dose of peanut protein increased from 0.1 to $800 \mathrm{mg}$ in eight months. Serum samples were drawn at baseline and after median 12 months of OIT build-up phase or avoidance. We analyzed serum IgG4 (30 $\mu 1$ of sample) in ImmunoCAP ISAC microarrays (ThermoFisher Scientific; Uppsala, Sweden). We considered the detection limit 0.1 ISU-G4 also as cut-off for positive response. The ethics committee of Helsinki University Hospital approved the study. One of the parents and the patient gave a written informed consent.

In analyzing repeated measures of the 112 allergens, we filtered the results so that in order to be included in the analysis an allergen had to show value above detection limit 0.1 (ISU-G4) in at least $50 \%+1$ of the patients in at least one of the four groups (pre OIT, post OIT, pre avoidance, post avoidance). We fitted a linear model to investigate the effects of treatment, response, subject, sex, and age. Furthermore, we used the eBayes test for pairwise comparisons of interest. P values were corrected in each pairwise comparison by using the Benjamini-Hochberg procedure. We used $\mathrm{R}$ program, version 3.0 (R Project for Statistical Computing, R Foundation, Vienna, Austria; http://www.r-project.org/) and IBM SPSS 22 (IBM, Armonk, NY).

Of the 39 patients on OIT, 34 achieved maintenance dosing, and 26/31 passed the double-blind placebo-controlled challenge with $1255 \mathrm{mg}$ peanut protein. Baseline characteristics and ISAC IgE levels are presented in the online supplemental table 1. IgG4 levels to Ara h 1, 2, and 6 increased during the treatment. Avoidance group showed no changes in IgG4 levels. (Figure 1) The ingested cumulative protein dose during the treatment was median $71 \mathrm{~g}$ (range $50 \mathrm{mg}$ to $158 \mathrm{~g}$ ). The cumulative protein dose correlated with the increase in $\mathrm{IgG} 4$ to Ara h 2 (rho 0.362, P=.024), but did not correlate with the IgG4 increase of

This article is protected by copyright. All rights reserved. 
Ara h 1 and Ara h 6.

The IgG4-increases for Ara h 1, 2, and 6 in the 26 patients who passed the final peanut challenge, i.e. desensitized patients, did not differ statistically from the five non-desensitized. (Data not shown)

Levels of other than peanut allergens that showed representative numbers of response are depicted in table 1, and other ISAC112 allergens in the online supplemental table 2.

To our knowledge this is the first study to examine microarrayed IgG4 profiles in patients who received peanut oral immunotherapy. During the treatment, the only IgG4 levels to change were peanut seed storage proteins Ara h 1,2, and 6. This is in line with our previous study were peanut OIT affected IgE only for the $2 \mathrm{~S}$ albumins Ara h 2 and 6. However, in the IgG4 antibodies, the effect included also a third seed storage protein, Ara h 1. Studies on peanut sensitization and allergy show that the 7S globulin Ara h 1 is more associated with peanut allergy than the $11 \mathrm{~S}$ globulin Ara h $3 .{ }^{7,8}$ In our previous study, IgG4, measured with ImmunoCAP, showed increases in Ara h 1 and Ara h 3, of which Ara h 1-change was stronger. ${ }^{5}$ In the present study, Ara h 3-response in IgG4 ISAC was underrepresented and therefore conclusions on effects on this allergen are limited. However, the response rate increased from $15 \%$ before OIT to $44 \%$ after OIT. (Table $1 \mathrm{~b}$ ) We believe that mild increase in Ara h 3 IgG4 in ISAC is possible.

The findings of unaffected Ara h 8- and Ara h 9- IgG4-levels are similar to our previous study on IgE microarray profiles in this patient population. ${ }^{5}$ Ara $\mathrm{h} 8$ is degraded in the gastric digestion and its' effects on IgG4 levels may be therefore limited. The amount of lipid transfer protein Ara h 9 is low in peanut ${ }^{9}$ which in turn may decrease the immunological effects of this stable allergen. Both Ara h 8and 9-IgG4 responses were infrequent in our study population.

Considering the development of tolerance, IgG4 may be the most important immunoglobulin in immunotherapy. Findings of this study further highlight the specificity of immunological response in peanut oral immunotherapy as IgG4 effects seem to occur only on the most peanut-specific allergens, but not on cross-reactive or unrelated allergens.

This article is protected by copyright. All rights reserved. 
Riikka Uotila, $M D^{a}$

Anna Kaarina Kukkonen, $M D, P h D^{a}$

Dario Greco, $P h D^{b}$

Anna S. Pelkonen, $M D, P h D^{a}$

Mika J. Mäkelä, MD, PhD ${ }^{a}$

From ${ }^{\text {a }}$ Allergology, University of Helsinki and Helsinki University Hospital; the Skin and Allergy Hospital

${ }^{\mathrm{b}}$ Faculty of Medicine and Life Sciences, Institute of Biosciences and Medical Technologies

(BioMediTech), University of Tampere and Institute of Biotechnology, University of Helsinki

\section{ACKNOWLEDGMENTS}

We acknowledge Mats Nystrand, Thomas Schlederer, and Malin Berthold from ThermoFisher Scientific for providing the ISAC IgG4 laboratory analyses. We received funding from the Foundation for Pediatric Research, the Sigrid Juselius Foundation, the Helsinki University Research Funds, the Foundation for Allergy Research, and the Helsinki Allergy Society. Prof Greco was supported by the Academy of Finland (grant agreements 275151 and 292307).

\section{ABBREVIATIONS}

Immuno-Solid phase Allergen Chip (ISAC)

ISAC standardized units (ISU)

Oral immunotherapy (OIT)

\section{REFERENCES}

(1) Burton OT, Logsdon SL, Zhou JS, Medina-Tamayo J, Abdel-Gadir A, Noval Rivas M, et al. Oral immunotherapy induces IgG antibodies that act through FcgammaRIIb to suppress IgE-mediated hypersensitivity. J Allergy Clin Immunol 2014;134:1310-1317.

(2) Jones SM, Pons L, Roberts JL, Scurlock AM, Perry TT, Kulis M, et al. Clinical efficacy and immune regulation with peanut oral immunotherapy. J Allergy Clin Immunol 2009;124:292-300.

This article is protected by copyright. All rights reserved. 
(3) Vickery BP, Lin J, Kulis M, Fu Z, Steele PH, Jones SM, et al. Peanut oral immunotherapy modifies IgE and IgG4 responses to major peanut allergens. J Allergy Clin Immunol 2013;131:12834.

(4) Kukkonen AK, Uotila R, Malmberg LP, Pelkonen AS, Mäkelä MJ. Double-blind placebocontrolled challenge showed that peanut oral immunotherapy was effective for severe allergy without negative effects on airway inflammation. Acta Paediatr 2017;106:274-281.

(5) Uotila R, Kukkonen AK, Greco D, Pelkonen AS, Mäkelä MJ. Peanut oral immunotherapy decreases IgE to Ara h 2 and Ara h 6 but does not enhance sensitization to cross-reactive allergens. J Allergy Clin Immunol 2017;139:1393-1396.

(6) Kukkonen AK, Pelkonen AS, Mäkinen-Kiljunen S, Voutilainen H, Mäkelä MJ. Ara h 2 and Ara 6 are the best predictors of severe peanut allergy: a double-blind placebo-controlled study. Allergy 2015;70:1239-1245.

(7) Ackerbauer D, Bublin M, Radauer C, Varga EM, Hafner C, Ebner C, et al. Component-resolved IgE profiles in Austrian patients with a convincing history of peanut allergy. Int Arch Allergy Immunol 2015;166:13-24.

(8) Agabriel C, Ghazouani O, Birnbaum J, Liabeuf V, Porri F, Gouitaa M, et al. Ara h 2 and Ara h 6 sensitization predicts peanut allergy in Mediterranean pediatric patients. Pediatr Allergy Immunol 2014;25:662-667.

(9) Krause S, Reese G, Randow S, Zennaro D, Quaratino D, Palazzo P, et al. Lipid transfer protein (Ara h 9) as a new peanut allergen relevant for a Mediterranean allergic population. J Allergy Clin Immunol 2009;124:771-778.

This article is protected by copyright. All rights reserved. 
a)

\begin{tabular}{|c|c|c|c|c|c|c|c|c|c|}
\hline OIT & Pre & & & Post & & & $\mathrm{P}$ & Pre & Post \\
\hline & Median & Min & Max & Median & Min & Max & & $\begin{array}{l}\text { No } \\
\text { resp }\end{array}$ & ents with \\
\hline Bet $\mathrm{v} 1$ & 0.32 & 0.05 & 3.6 & 0.46 & 0.05 & 3.1 & .65 & 30 & 31 \\
\hline Mal d 1 & 0.10 & 0.05 & 2.4 & 0.12 & 0.05 & 2.1 & .85 & 20 & 22 \\
\hline Bos d 8 & 0.50 & 0.05 & 11.4 & 2.3 & 0.05 & 22.4 & .85 & 28 & 30 \\
\hline Bos d 5 & 2.1 & 0.05 & 17.6 & 2.4 & 0.05 & 18.8 & .85 & 36 & 36 \\
\hline Bos d 4 & 2.1 & 0.05 & 21.6 & 0.48 & 0.05 & 13.7 & .85 & 28 & 27 \\
\hline $\begin{array}{l}\text { Bos d } \\
\text { Lactoferrin }\end{array}$ & 0.56 & 0.05 & 5.9 & 0.50 & 0.05 & 8.0 & .85 & 28 & 30 \\
\hline Gal d 1 & 0.88 & 0.05 & 19.6 & 1.1 & 0.05 & 22.6 & .85 & 32 & 34 \\
\hline Gal d 2 & 1.8 & 0.05 & 13.3 & 2.2 & 0.05 & 11.9 & .85 & 33 & 32 \\
\hline Gal d 3 & 0.12 & 0.05 & 0.80 & 0.14 & 0.05 & 0.70 & .85 & 21 & 25 \\
\hline Tri a aA_TI & 0.28 & 0.05 & 2.9 & 0.26 & 0.05 & 3.2 & .85 & 25 & 26 \\
\hline Tri a 14 & 0.05 & 0.05 & 9.5 & 0.10 & 0.05 & 14.4 & .85 & 19 & 21 \\
\hline Avoidance & Pre & & & Post & & & $\begin{array}{l}\mathrm{P} \\
\text { value }\end{array}$ & Pre & Post \\
\hline & Median & Min & Max & Median & Min & Max & & $\begin{array}{l}\text { No } \\
\text { resp }\end{array}$ & ents with \\
\hline Bet v 1 & 0.26 & 0.05 & 14.1 & 0.16 & 0.05 & 0.90 & 1 & 15 & 12 \\
\hline Mal d 1 & 0.12 & 0.05 & 2.4 & 0.12 & 0.05 & 1.0 & 1 & 11 & 10 \\
\hline Bos d 8 & 0.74 & 0.05 & 31.0 & 0.32 & 0.05 & 27.9 & 1 & 19 & 17 \\
\hline Bos d 5 & 3.7 & 0.05 & 25.1 & 2.0 & 0.05 & 19.2 & 1 & 19 & 17 \\
\hline Bos d 4 & 3.7 & 0.05 & 28.9 & 2.7 & 0.05 & 23.5 & 1 & 19 & 17 \\
\hline $\begin{array}{l}\text { Bos d } \\
\text { Lactoferrin }\end{array}$ & 0.47 & 0.05 & 1.9 & 0.55 & 0.05 & 1.8 & 1 & 17 & 15 \\
\hline Gal d 1 & 1.5 & 0.05 & 13.6 & 1.6 & 0.05 & 18.1 & 1 & 18 & 16 \\
\hline Gal d 2 & 3.7 & 0.05 & 19.8 & 2.5 & 0.05 & 24.4 & 1 & 19 & 17 \\
\hline Gal d 3 & 0.24 & 0.05 & 1.2 & 0.16 & 0.05 & 1.4 & 1 & 16 & 14 \\
\hline Tri a aA_TI & 0.20 & 0.05 & 2.4 & 0.14 & 0.05 & 6.4 & 1 & 16 & 14 \\
\hline Tri a 14 & 0.18 & 0.05 & 5.7 & 0.05 & 0.05 & 26.2 & 1 & 14 & 8 \\
\hline
\end{tabular}

This article is protected by copyright. All rights reserved. 
b)

\begin{tabular}{|c|c|c|c|c|}
\hline \multicolumn{3}{|l|}{ OIT } & \multicolumn{2}{|l|}{ Avoidance } \\
\hline & $\begin{array}{l}\text { Pre, n } \\
(\%)\end{array}$ & $\begin{array}{l}\text { Post, } \mathrm{n} \\
(\%)\end{array}$ & $\begin{array}{l}\text { Pre, n } \\
(\%)\end{array}$ & $\begin{array}{l}\text { Post, } \mathrm{n} \\
\text { (\%) }\end{array}$ \\
\hline Ara h 1 & $7(18)$ & 27 (69) & $8(44)$ & $6(33)$ \\
\hline Ara h 2 & $15(38)$ & $37(95)$ & $11(61)$ & 7 (39) \\
\hline Ara h 3 & $6(15)$ & $17(44)$ & $5(28)$ & $3(17)$ \\
\hline Ara h 6 & $16(41)$ & $37(95)$ & $11(61)$ & $10(56)$ \\
\hline Ara h 8 & 7 (18) & $10(26)$ & $2(11)$ & 0 \\
\hline Ara h 9 & $12(31)$ & $12(31)$ & $3(17)$ & $4(22)$ \\
\hline
\end{tabular}

Table 1. a) IgG4 levels and number of patients with response (ISU-G4 $\geq 0.1$ ) before and after treatment in oral immunotherapy $(\mathrm{OIT})(\mathrm{n}=39)$ and avoidance $(\mathrm{n}=18)$ groups. Allergens that showed representative response are included. (see methods section) b) Number of patients with IgG4 response (ISU-G4 $\geq 0.1$ ) for peanut allergens in immunotherapy and avoidance groups.

This article is protected by copyright. All rights reserved. 


\section{Figure and supplementary table legends:}

Figure 1. Levels of $\operatorname{IgG} 4$ to peanut allergens in a) oral immunotherapy group $(n=39)$ and $b)$ avoidance group $(n=18)$ before and after treatment. Ara h 3, Ara h 8, and Ara h 9 are excluded from the statistical analysis due to limited number of responses. (see Methods section)

Supplemental table online 1. Baseline characteristics of patients in OIT $(n=39)$ and avoidance $(n=18)$ groups.

Supplemental table online 2. Levels of IgG4 and number of patients with response (ISU-G4 $\geq 0.1$ ) before and after peanut oral immunotherapy. Allergens with no response in any of the patients are excluded from the table (Amb a 1, Ani s 1, Ani s 3, Api m 1, Art v 1, Asp f 3, Asp f 6, Ber e 1, Bet v 4, Bla g 7, Blo t 5, Che a 1, Cla h 8, Der f 2, Der p 1, Der p 2, Fag e 2, Gal d 5, Hev b 1, Hev b 3, Hev b 5, Hev b 6.01, Ole e 1, Pen m 2, Phl p 11, Phl p 12, Phl p 2, Phl p 7, Pla a 1, Pla 1 1, Pol d 5, Tri a 19.0101, and Ves v 5.).

This article is protected by copyright. All rights reserved. 


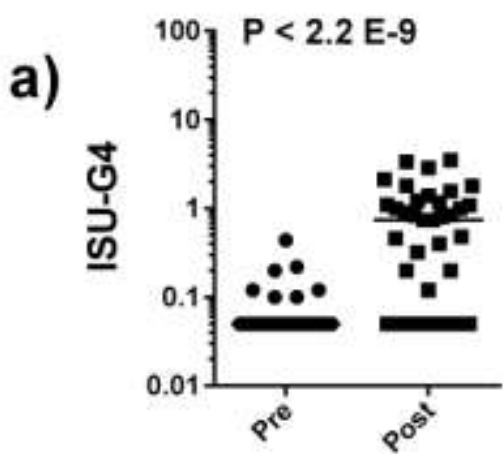

Ara $\mathbf{h} 1$

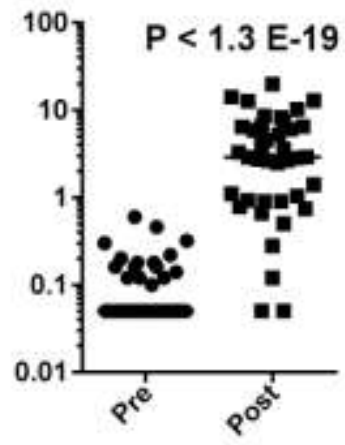

Ara h 6

b)

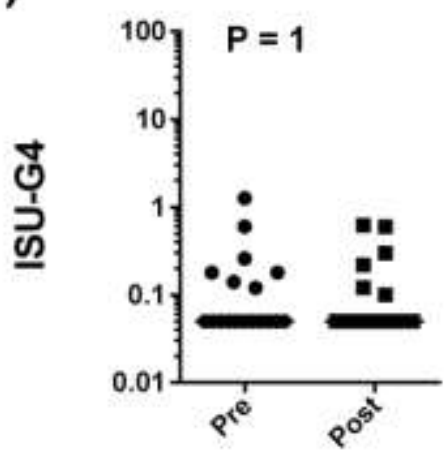

Ara h 1

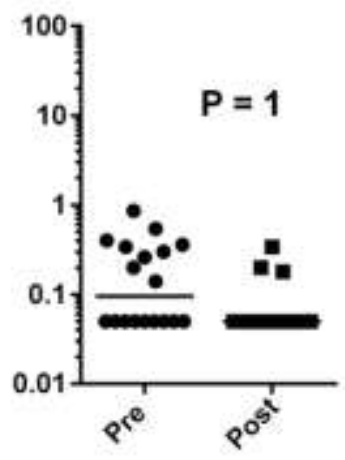

Ara h 6

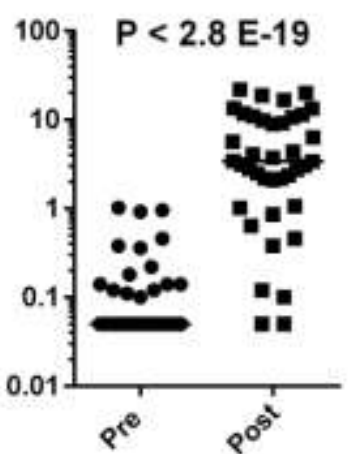

Ara h 2

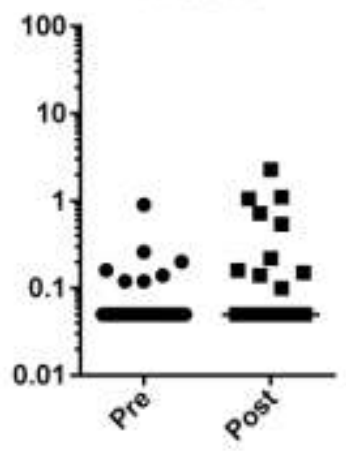

Ara h 8

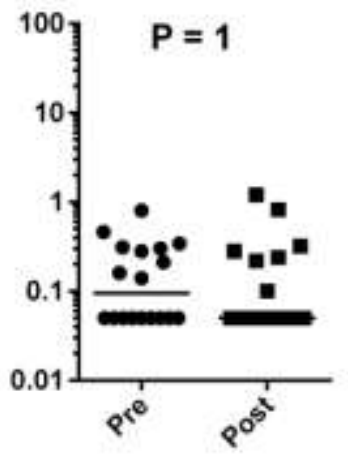

Ara h 2

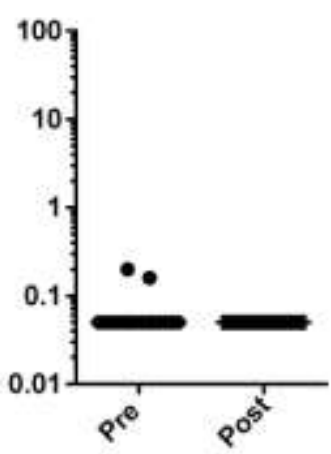

Ara h 8

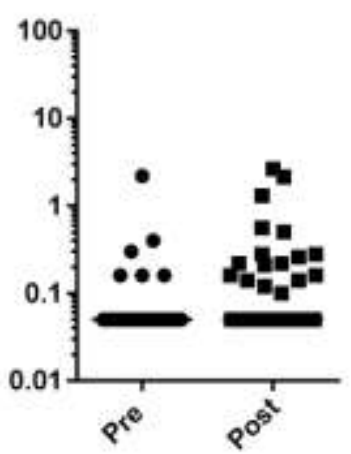

Ara h 3

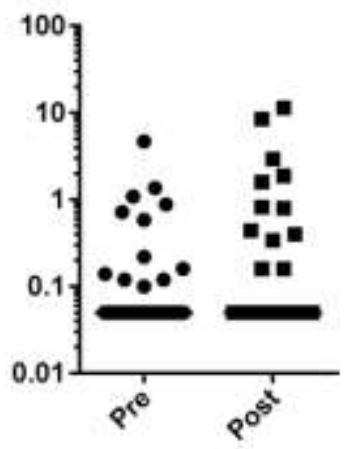

Ara h 9

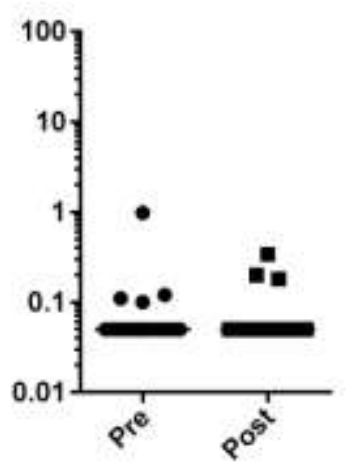

Ara h 3

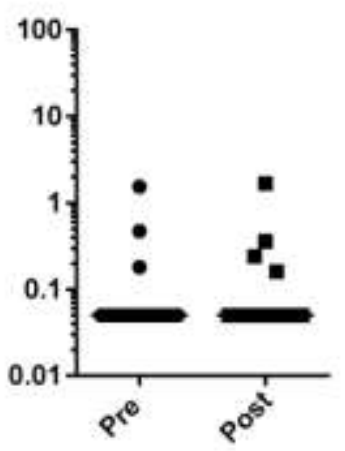

Ara h 9 